\title{
Ring chromosome 21 syndrome
}

INSERM

\section{Source}

INSERM. (1999). Orphanet: an online rare disease and orphan drug data base. Ring chromosome 21 syndrome. ORPHA:1445

Ring chromosome 21 syndrome is an autosomal anomaly characterized by variable clinical features, most commonly including growth retardation, developmental delay, intellectual disability, epilepsy, microcephaly, short stature, dysmorphic features, hypog ammag lobulinemia, thrombocytopenia and unspecific skeletal anomalies (hemivertebrae, clinodactyly, syndactyly). In rare cases, it has been described in phenotypically normal individuals. 